\section{Intersections}

Canadian Journal of Music

Revue canadienne de musique
Intersections CANADIAN JOURAL OR MUSIO

\title{
La musique dans la vie des jeunes enfants : recension, analyse et critique de quelques théories du développement musical
}

\section{Jonathan Bolduc}

Volume 27, numéro 2, 2007

URI : https://id.erudit.org/iderudit/1013111ar

DOI : https://doi.org/10.7202/1013111ar

Aller au sommaire du numéro

Éditeur(s)

Canadian University Music Society / Société de musique des universités canadiennes

ISSN

1911-0146 (imprimé)

1918-512X (numérique)

Découvrir la revue

Citer cet article

Bolduc, J. (2007). La musique dans la vie des jeunes enfants : recension, analyse et critique de quelques théories du développement musical. Intersections,

27(2), 19-35. https://doi.org/10.7202/1013111ar
Résumé de l'article

Les recherches concernant le développement des capacités musicales chez les jeunes enfants sont en plein essor. Inspirés par différentes théories constructivistes et socioconstructivistes, quelques psychologues de la musique ont, depuis les 30 dernières années, élaboré différents modèles afin d'éclairer les savoirs musicaux présents à la période préscolaire. Cet article recense, analyse et critique cinq des principaux modèles qui ont été développés au cours de ces trois décennies. Il réitère également l’importance qui doit être accordée à l'éducation musicale au cours de la petite enfance, et du rôle positif quelle peut jouer dans le cadre d'un enseignement interdisciplinaire.
Copyright @ Canadian University Music Society / Société de musique des universités canadiennes, 2008
Ce document est protégé par la loi sur le droit d'auteur. L'utilisation des services d'Érudit (y compris la reproduction) est assujettie à sa politique d'utilisation que vous pouvez consulter en ligne.

https://apropos.erudit.org/fr/usagers/politique-dutilisation/ 


\section{LA MUSIQUE DANS LA VIE DES JEUNES ENFANTS : RECENSION, ANALYSE ET CRITIQUE DE QUELQUES THÉORIES DU DÉVELOPPEMENT MUSICAL}

\section{Jonathan Bolduc}

\section{INTRODUCTION}

La musique occupe une place importante dans la vie de tous les enfants, et ce, même avant leur naissance. Des études révèlent que durant la grossesse, le fœtus réagit déjà aux paroles et aux chansons qui lui sont familières (Hepper 1991 ; Woodward 1992). Différentes équipes de chercheurs ont aussi constaté que dès son jeune âge, le nourrisson possède des connaissances musicales innées et acquises qui lui permettent, entre autres, de distinguer des séquences mélodiques consonantes et dissonantes (Trehub 2001) ainsi que de reconnaître des enchâ̂nements rythmiques semblables et différents (Thorpe, Trehub, Morrongiello et Bull 1988).

Malgré les progrès de la psychologie depuis la fin des années 1960, plusieurs chercheurs prétendent que le développement musical demeure énigmatique et qu'un nombre limité de modèles permet d'expliquer en partie comment le jeune enfant développe ses capacités musicales (pour une synthèse, voir Flohr 2003). Dans le but d'éclairer la façon dont les enfants d'âge préscolaire construisent leurs savoirs musicaux, cet article recense, analyse et critique cinq modèles du développement musical élaborés depuis les trois dernières décennies. D’entrée de jeu, nous examinerons sommairement divers critères d'évaluation permettant de juger de la validité et de la pertinence d'une théorie du développement sur les plans conceptuel et méthodologique. Par la suite, nous résumerons les principaux fondements des théories constructiviste de Piaget et socioconstructiviste de Vygotsky, considérant l'impact majeur que les travaux de ces deux chercheurs ont eu auprès de nombreux psychologues de la musique. Enfin, cinq modèles du développement musical, soit ceux de Sloboda (1985), Swanwick et Tillmann (1986), Zenatti $(1967,1990)$, Gordon $(1988,2003)$ et Hargreaves (1995) seront approfondis. En guise de conclusion, nous réitérerons l'importance qui doit être accordée à l'éducation musicale au cours de la petite enfance et le rôle positif qu'elle peut jouer dans le cadre d'un enseignement interdisciplinaire.

\section{LES CRITÈRES D'ÉVALUATION D'UNE THÉORIE DU DÉVELOPPEMENT}

Selon Green et Piel (1989), cinq critères permettent de juger de la valeur scientifique d'une théorie du développement sur les plans conceptuel et méthodo- 
logique. D’abord, une théorie développementale doit être vérifiable auprès de différentes populations (ce que les chercheurs désignent par le terme de "testability»). De plus, sa capacité de décrire la réalité doit s’appuyer sur des recherches empiriques, sur une forme de validité externe (external validity). Elle est tenue de prévoir une série de comportements observables et prédictifs à des périodes précises, plus particulièrement durant la petite enfance et l'enfance (predictive validity). L'ensemble de ces fondements doit être uni d'une façon logique afin de montrer sa cohérence interne (internal consistency). Enfin, une théorie développementale doit être facilement compréhensible et posséder un nombre limité de concepts (ce que les chercheurs nomment " theoretical economy»).

Hargreaves et Zimmermann (1992) et Swanwick (2001) estiment que les critères énumérés par Green et Piel (1989) sont essentiels dans toutes les théories développementales, mais ils leurs adjoignent deux autres propres au domaine musical ${ }^{1}$. D'une part, un modèle du développement musical doit être global et considérer à la fois les paramètres musicaux (hauteur, durée, intensité, timbre) et les paramètres extra musicaux (capacités d’écoute active, de création musicale, d'interprétation musicale, etc.). D'autre part, il doit tenir compte du capital biologique de l'individu et de son environnement d'apprentissage. Ce second critère est directement inspiré des théories constructiviste de Piaget (1977) et socioconstructiviste de Vygotsky (1978), bien quaucun de ces deux chercheurs ne se soit intéressé précisément au développement musical.

\section{Les influences de Piaget et de Vygotsky}

Les travaux de Piaget $(1971,1977,1978,1984,2003)$ nous renseignent principalement sur le développement intellectuel de l'enfant. Le chercheur est d'avis que l'intelligence se construit par l'équilibration des structures cognitives (schèmes), c'est-à-dire une adaptation progressive de l'enfant à son environnement. L’apprenant intègre d'abord les éléments du milieu (intégration du réel à soi), puis il les modifie pour s'ajuster à de nouvelles expériences (ajustement de soi au réel). Ces deux mécanismes fondamentaux, nommés assimilation et accommodation, gouvernent la construction des connaissances chez lenfant. En opposition à Vygotsky (1978), qui privilégie la coopération sociale dans l'apprentissage, Piaget $(1971,1977)$ prétend que l'enfant apprend par lui-même et que le soutien accordé par un individu plus expérimenté peut contraindre son développement. Il dit d'ailleurs que « tout ce quon apprend à l'enfant, on l'empêche de l'inventer ou de le découvrir ${ }^{2}$ "»

Dans la théorie piagétienne, l'enfant franchit quatre stades cognitifs, de la naissance jusquà l'adolescence. Le stade sensori-moteur correspond aux deux premières années de vie; le stade préopératoire est associé à la période entre lâge de deux et sept ans; le stade des opérations concrètes représente celle de sept à 11 ans alors que le stade des opérations formelles équivaut à la période de

1 Les cinq modèles de développement musical présentés dans cet article tiennent compte de ces deux critères supplémentaires mis de l'avant par Hargreaves et Zimmermann (1992) ainsi que Swanwick (2001).

2 D’après les propos recueillis par Bringuier (1977). 
12 à 16 ans environ. Même si un âge approximatif est proposé pour chacun de ces stades, Piaget et Inhelder (2003) croient que l'enfant y progresse à son propre rythme. Il peut être en chevauchement lorsqu'il s'engage dans un stade cognitif supérieur sans avoir complètement assimilé les notions du précédent.

Piaget et Inhelder (2003) remarquent que l'ensemble des enfants d'âge préscolaire atteint le stade préopératoire. Ce stade est caractérisé par l'émergence de la fonction symbolique, c'est-à-dire la possibilité de représenter un "signifié " par un "signifiant " qui lui est propre (Cloutier, Gosselin et Tap, 2004). L'apparition de la représentation mentale amène donc l'enfant à reconstruire sur le plan de la pensée ce qu'il a acquis sur le plan de l'action au stade sensori-moteur. Cette équilibration s'effectue, entre autres, par l'imitation et le jeu symbolique. Le dessin et l'imagerie mentale sont aussi deux autres moyens par lesquels l'enfant rend compte des actions intériorisées. En effet, l'enfant est capable de se représenter mentalement des objets ou des événements à l'aide de symboles. Sa pensée demeure toutefois statique et non réversible, en ce sens qu'il lui manque la mobilité permettant les opérations, qui sont des actions intériorisées. Notons, finalement, que le langage connait aussi un essor important à ce stade. Piaget (1984) lui confère essentiellement un rôle égocentrique et le considère comme secondaire dans l'apprentissage. La position qu'il adopte est souvent critiquée, étant donné qu'il néglige l'influence des facteurs sociaux et culturels dans le développement de l'enfant.

Pour sa part, Vygotsky (1978) estime que les fonctions mentales supérieures ne peuvent se développer sans l'apport constructif des interactions sociales ${ }^{3}$. Selon lui, les apprentissages de l'enfant d'âge préscolaire se font d'abord avec l'aide d'autrui avant dêtre réalisés d'une façon autonome. La période séparant ces deux étapes est nommée "zone proximale de développement » et représente la différence exprimée en unités de temps entre le passage de l'interpsychique à l'intrapsychique (Rieber, Robinson et Bruner 2004). Dans cette théorie, l'apprentissage gouverne le développement et s'effectue d'une façon continue et graduelle.

D’après le chercheur russe, la place accordée au développement linguistique montre à quel point celui-ci joue un rôle crucial dès les premières années de vie (Ivic 1994). C'est en communiquant avec d'autres individus que l'enfant affine ses connaissances et en acquiert de nouvelles. À lâge préscolaire, l'apprenant utilise aussi le langage pour " communiquer avec lui-même ". Le langage intérieur permet d’éclairer les stratégies employées par l'enfant et la façon dont il planifie ses activités. Il offre aussi la chance d'examiner comment il transforme les commentaires formulés par l'adulte lorsqu'il se trouve face à un nouveau dilemme. Ces échanges communicatifs s'avèrent nécessaires au développement de la pensée.

3 Vygotsky (1978) distingue les fonctions mentales élémentaires des fonctions mentales supérieures en affirmant que les secondes impliquent 1) la régulation volontaire de l'activité; 2) la prise de conscience de ses propres processus mentaux; 3 ) la considération de lorigine et de la nature sociales des fonctions mentales supérieures; 4) l'utilisation de signes servant à médiatiser les fonctions mentales supérieures (Legendre 2005, 356). 
Lattention accordée à la culture dans le développement de l'enfant est l'aspect principal qui distingue Vygotsky de Piaget (Ivic 1994). Pour Vygotsky (1978), l'apprentissage représente un processus d'acculturation, au sens où l'enfant intègre les savoirs en regard de leur utilisation contextuelle. Comme l'explique Legendre,

on ne peut isoler les connaissances des divers contextes dans lesquels elles sélaborent. [...] La cognition est socialement située, lactivité mentale ne pouvant être dissociée de lenvironnement culturel et social dans laquelle elle se réalise et des ressources que celui-ci met à sa disposition. $(2005,364)$

Selon la théorie vygotskienne, la culture influence nos manières de penser et soutient le développement des fonctions mentales supérieures. C'est grâce au développement de ces dernières que l'enfant parvient à s'intégrer dans une communauté.

Il parait inévitable de s'inspirer des travaux de ces deux chercheurs afin d'expliciter le développement musical de l'enfant. Toutefois, un simple transfert des concepts piagétiens et vygotskiens en musique ne rend pas compte de la spécificité du domaine. Des modèles propres au développement musical ont donc été élaborés. Les cinq principaux modèles développés au cours des 30 dernières années sont présentés ci-dessous.

\section{LE MOdÈLE DE SLOBODA}

Sloboda (1985) s'appuie sur deux théories constructivistes pour expliquer le développement musical de l'enfant. Suivant la théorie piagétienne, il considère que chaque individu progresse à travers une série de stades cognitifs. Le développement musical est caractérisé par l'assimilation de schèmes et par l'accommodation qui permet de restructurer les schèmes mentaux incompatibles avec la réalité (Piaget et Inhelder 2003). L'interprétation de Piaget par Sloboda laisse supposer une conception similaire du développement musical ; l'observation de comportements musicaux d'enfants à des âges précis rend compte de leurs capacités cognitives pour une période donnée.

Pour Sloboda (1985), à l'instar de Chomsky $(1957,1965)$, chaque individu possède en outre des aptitudes qui peuvent l'amener à exceller dans un nombre limité de capacités cognitives, telles que le langage ou la musique. Il est possible que des zones cérébrales soient consacrées exclusivement à la musique et qu'elles permettent à l'individu de développer des capacités propres à ce domaine. Une telle hypothèse reconnaît un ancrage biologique à la musique, ce qui signifie que les enfants font preuve d'une compréhension précoce de certains phénomènes musicaux, même avant leur naissance (Ilari 2002).

Sloboda (1985) présente deux types de comportements caractéristiques du développement musical de l'enfant d'âge préscolaire. D'une part, il affirme que l'enfant âgé de quatre à six ans se situe dans une phase "imitative ". Sur le plan musical, lapprenant tente d'abord de reproduire les paroles de chansons, ensuite des fragments mélodiques ou rythmiques et enfin, des chansons complètes (Moog 1976). L'enfant est sensible à la reproduction exacte et ce besoin de vouloir 
imiter fidèlement un événement (sonore ou autre) se retrouve dans d'autres domaines symboliques de son développement, tels que le dessin (Wallon 1996).

D'autre part, lenfant d'âge préscolaire s'engage progressivement dans un processus de création. Il s'initie à des activités d'improvisation instrumentale ou vocale non structurées pour ensuite intégrer des éléments propres à sa culture musicale. Sloboda prétend que l'enfant utilise généralement des intervalles diatoniques dans la création de ses productions instrumentales et emploie des phrases provenant de chansons connues dans la création de ses productions vocales. Sur ce dernier point, le chercheur fait remarquer que la maîtrise progressive des règles de la tonalité et de la métrique par l'enfant guide la réalisation de ses chansons. Sa connaissance des structures harmoniques est toutefois rudimentaire.

Selon Sloboda, les facteurs innés et environnementaux jouent un rôle crucial dans le développement musical dès la petite enfance. Il considère que " la compétence musicale s'acquiert à la faveur d'une interaction avec un environnement musical et que [...] tout progrès humain implique une construction au départ de ce qui existe déjà » (Sloboda 1985, 265). D’après lui, le développement musical se réalise en deux phases. La première phase, nommée acculturation musicale, correspond à la sensibilisation musicale au cours des dix premières années de vie 4 . Nous pouvons y voir un rapprochement avec la théorie vygotskienne, même si Sloboda ne s'y réfère pas directement. La seconde phase, appelée formation et acquisition de compétences, renvoie, pour sa part, à l'analyse des comportements musicaux à une période plus tardive, soit à l'adolescence et au début de lâge adulte.

L'apport de Sloboda au domaine de la psychologie de la musique est incontestable, si nous considérons le fait quau milieu des années 1980, peu de chercheurs avaient étudié spécifiquement le développement musical de l'enfant d'âge préscolaire (Fraisse 1974; Francès 1984; Gordon 1971, 1979; Imberty 1969; Pflederer 1964; Zenatti 1967). Néanmoins, son interprétation des concepts de Piaget $(1971,1984,2003)$ et de Chomsky $(1957,1965)$ en musique est parfois critiquée. On lui reproche d’établir a priori les « grandes lignes » du développement musical sans avoir examiné les comportements musicaux chez les enfants. Sloboda (1985) se défend en affirmant que la théorie piagétienne, abondamment documentée, présuppose des conduites musicales chez tous les individus. Pour sa part, la théorie chomskienne a fait valoir, à maintes reprises, l'importance du capital biologique dans l'apprentissage. D'autres auteurs et chercheurs ont d'ailleurs souligné la pertinence de ce dernier aspect en musique (Bernstein 1976; Lowe 1995, 1998). Bien que le modèle développemental de Sloboda soit remis en cause, certains de ses fondements ont inspiré lélaboration des théories subséquentes.

4 Selon Chauveau, le processus d'acculturation, ou d'intégration culturelle, comporte deux processus « il y a simultanément processus d'extériorisation (s'intégrer dans, entrer dans, s'assimiler à [...]) et processus d'intériorisation (intégrer, faire entrer en soi, assimiler les pratiques [...]) » $(1997,156)$. L'enfant doit entrer dans le monde de la musique, le découvrir, le fréquenter et le pratiquer, mais aussi le faire entrer en soi, le transformer en attitudes, en mode de pensée et en dispositions réflexives personnelles. (Adaptation des propos de l'auteur) 


\section{Le Modèle De Swanwick ET TillmanN}

Le modèle de Swanwick et Tillmann (1986) s'inspire en grande partie des travaux de Piaget $(1971,2003)$ et de Piaget et Inhelder (2003). Il est représenté par la forme d'une spirale comprenant quatre boucles développementales, de la petite enfance à l'adolescence. Le modèle est basé sur trois principes organisateurs, soit les types d'activités, les phénomènes musicaux et les expériences du développement (Zulauf 2002).

En premier lieu, chaque phase du développement musical correspond à une boucle de la spirale à laquelle sont associées des activités précises. Durant la petite enfance (0-4 ans) l'enfant découvre et manipule les paramètres sonores. La période de l'enfance (4-10 ans), celle qui nous intéresse dans cet article, est caractérisée par les jeux imitatifs alors que l'enfant tente de représenter musicalement le monde qui lentoure. La préadolescence (10-15 ans) correspond à la pratique des jeux imitatifs. À ce moment, l'apprenant continue son apprentissage musical et s'engage dans un processus de création plus complexe, entamé au cours de l'enfance. Durant la quatrième période, nommée métacognitive, l'adolescent (15 ans et plus) prend conscience de ses propres processus de pensée et rend compte de ses expériences sur le plan musical.

En deuxième lieu, l'arrière de chaque boucle de la spirale (section ombragée) indique des phénomènes musicaux propres à chacun des groupes dâge. Les phénomènes précisent les aspects sur lesquels l'enfant centre successivement son attention au cours de son développement musical. La période de la petite enfance est caractérisée par les matériaux sonores, celle de l'enfance par l'expression, celle de la préadolescence par la forme et celle de ladolescence par la valeur de la musique. Swanwick et Tillmann considèrent les deux premières périodes comme des phases de sensibilisation et d'exploration, tandis que les deux dernières représentent des phases d'appropriation.

En troisième lieu, les quatre boucles de la spirale regroupent une série d'expériences musicales individuelles (associées à la partie gauche de la spirale) et une série d'expériences musicales sociales (associées au côté droit de la spirale). Comme le souligne Zulauf, " ce principe organisateur ferait passer l'elève d'une expérience personnelle vers des formes plus socialisées et schématiques. Chaque niveau se voit ainsi subdivisé en deux, créant [...] huit modes développementaux " $(2002,35)$. Pour la période de l'enfance, nous remarquons que lenfant s'engage d'abord dans des activités d'expression personnelle en tentant d'exprimer sa propre réalité du monde par la musique. Par la suite, ces dernières activités font place à des activités coopératives où l'apprenant travaille davantage en collaboration avec ses pairs afin de s'engager dans un processus de création musicale plus complexe.

Même si Swanwick et Tillmann (1986) ont établi une genèse du développement musical, certains chercheurs relèvent des faiblesses dans leur modèle sur les plans conceptuel et méthodologique (Hargreaves et Zimmermann 1992; Zulauf 2002). D’une part, ils contestent la fidélité des deux auteurs à la terminologie piagétienne; les psychologues critiquent notamment une application boiteuse des concepts d'assimilation et d'accommodation. Swanwick (2001) clarifiera cette question quelques années plus tard en spécifiant que l'assimilation 
correspond au côté gauche de la spirale alors que l'accommodation est associée au côté droit, se rapprochant ainsi des idées de Piaget.

D'autre part, Swanwick et Tillmann (1986) ont recueilli un nombre important de productions musicales d'enfants afin d'établir les fondements de leur modèle ( 745 productions vocales et instrumentales réalisées par 48 enfants). Ces productions ont été réalisées uniquement par des sujets âgés de trois à neuf ans. En s'appuyant sur d’autres études (Moog 1976, entre autres), Swanwick et Tillmann ont extrapolé leur modèle sans mener de recherches empiriques pour les périodes de la préadolescence et de l'adolescence. Dès lors, des chercheurs s'interrogent sur la validité de ce modèle pour les sujets âgés de 10 ans et plus (Hargreaves et Zimmerman 1992; Zulauf 2002). Ils admettent néanmoins que le modèle en spirale des deux auteurs offre une représentation convaincante du développement des capacités musicales durant l'enfance et qu'il éclaire le développement musical à lâge préscolaire.

Figure 1. Modèle du développement musical de Swanwick et Tillmann (1986)

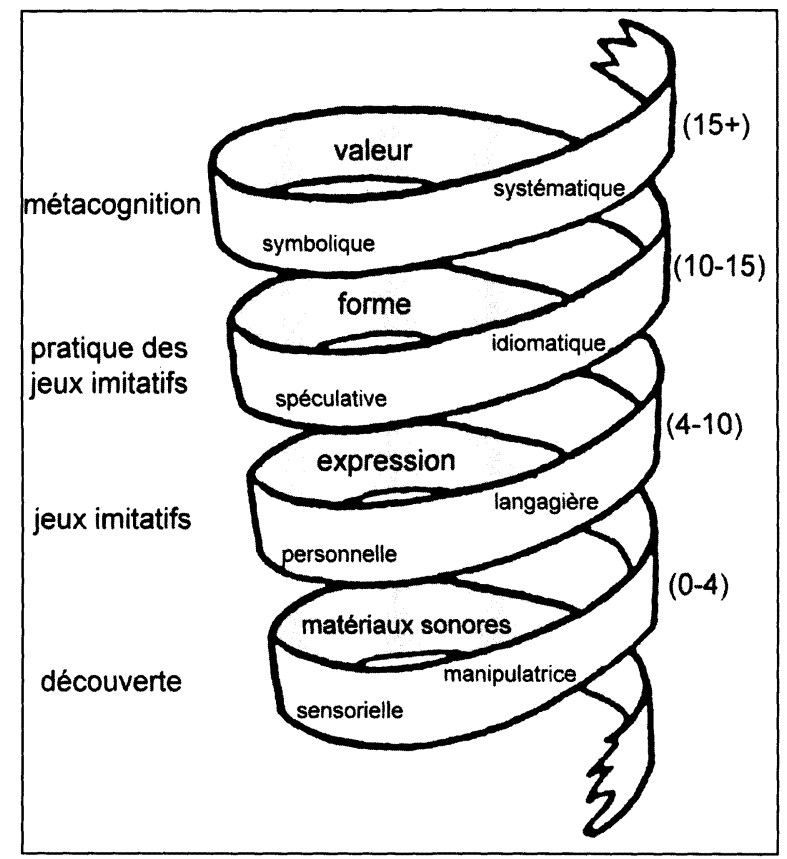

\section{LE MODÈLE DE ZENATTI}

Inspirée par les travaux de Piaget (1961) sur les mécanismes de perception visuelle, Zenatti $(1967,1990)$ s'est principalement intéressée à la perception musicale. Selon elle, le développement des capacités musicales - quelle nomme la genèse de l'intelligence musicale - s'effectue en trois phases. L'individu acquiert d'abord le sens de l'intervalle au cours de la petite enfance (0-4 ans), il organise ensuite les intervalles en système durant l'enfance (5-8 ans) et enfin, il acquiert 
le sens de l'organisation d'une œuvre musicale (âge indéterminé). Zenatti (1967, 1990) soutient que, « toute acquisition se fait d'abord de manière perceptive, par une sorte d'imprégnation, avant que l'enfant ne soit capable de l'utiliser dans ses productions, d'une manière [...] "opérative" " (Zulauf 2002, 28-29).

$\mathrm{Au}$ cours de la première phase du modèle de Zenatti (l'acquisition du sens de l'intervalle), l'enfant reconnait tout d'abord un certain nombre de mélodies propres à sa culture. La phase atteint son point d'équilibre lorsque l'enfant parvient à reproduire vocalement ces mélodies (imitation vocale vers lâge de 15 mois) ou à les jouer sur un instrument de musique (imitation instrumentale entre lâge de deux à quatre ans). Selon la chercheuse, l'accomplissement d'activités musicales reproductives par le jeune enfant dénote une compréhension primaire de lorganisation musicale et un processus d'assimilation de la hiérarchie du système tonal en cours.

La deuxième phase consiste en "l'assimilation de la hiérarchie interne à la suprématie de certains degrés de l'échelle tonale " (Zenatti 1990, 30). Sur le plan perceptif, l'enfant préfère les mélodies se terminant par la tonique et devient plus attentif aux différentes cadences (suspensives ou conclusives). D'après la chercheuse, le point d'équilibre de cette seconde phase est atteint au moment où l'enfant réussit, par lui-même, à organiser les sons musicaux selon l'échelle tonale. La compréhension des phénomènes musicaux se manifeste, entre autres, à travers les improvisations vocales et instrumentales. Entre cinq et huit ans, la durée des chants spontanés et des compositions musicales de l'enfant s'accroît et ce dernier respecte davantage le centre tonal ${ }^{5}$. Les intervalles qu'il utilise se précisent et s'apparentent aux modèles favorisés par l'adulte (Ribière-Raverlat 1997, Zenatti 1990).

Au cours de la troisième phase (qui correspond à l'acquisition de l'organisation de l'œuvre musicale), l'individu parvient à analyser les aspects mélodiques, rythmiques, harmoniques et polyphoniques d'une œuvre. Toutefois, étant donné son degré d'abstraction, cette phase est franchie par un nombre limité de gens qui possèdent généralement des connaissances approfondies en musique. Elle sous-entend l'interprétation et la composition musicales expertes.

Les travaux de Zenatti $(1967,1976,1990)$ nous fournissent quelques précisions concernant le développement musical de l'enfant. La chercheuse prétend que l'intelligence musicale se construit graduellement grâce à une interaction entre les activités perceptives et les activités sensori-motrices, ce qui permet de coordonner les éléments musicaux. Favorisant une approche développementale constructiviste, Zenatti $(1967,1990)$ considère, à l'instar de l'ensemble des travaux de Piaget, que l'enfant est agent actif de son développement et qu'il acquiert ses capacités musicales par léquilibration des structures cognitives.

5 Le centre tonal correspond à l'ensemble des relations entre les degrés hiérarchisés d'une échelle musicale donnée par rapport à son degré fondamental, la tonique (Deliège et Sloboda 1995). 


\section{LE MODÈLE DE GORDON}

Gordon $(1988,2003)$ a élaboré un modèle socioconstructiviste visant à expliquer le développement des aptitudes musicales ${ }^{6}$. Selon lui, ces dernières sont à la fois une combinaison du potentiel inné et des expériences sociales vécues au cours de la petite enfance. Le degré d'aptitude musicale que possède chaque individu est unique et ne peut pas être déterminé seulement par des facteurs héréditaires (Gordon, 1999). Gordon prétend que le potentiel musical est à son apogée à la naissance. Si l'enfant n'est pas stimulé par des activités musicales au cours des premières années de sa vie (0-6 ans), ses aptitudes musicales innées s'atrophient graduellement. Le chercheur a remarqué que le potentiel musical diminue surtout entre lâge de quatre à six ans pour se stabiliser vers l'âge de neuf ans. Il considère donc que la période préscolaire est essentielle à la mobilisation des aptitudes musicales.

Gordon (2003) présente trois stades préparatoires dans lesquels s'intègrent sept phases développementales propres aux enfants âgés de zéro à six ans. Ce regroupement, nommé types and stages of preparatory audiation ${ }^{7}$, précède les six stages of audiation de la music learning theory, consacrés à une population âgée de six à 15 ans. Le chercheur considère que le développement musical s'effectue par phases et que chaque personne les franchit à son propre rythme. Certains enfants progressent plus rapidement que d'autres, étant donné leur niveau d'aptitudes musicales innées plus élevé. Comme Piaget et Inhelder (2003), Gordon (1999) mentionne également qu'un enfant peut être en chevauchement lorsqu'il commence son apprentissage dans un stade supérieur sans maîtriser toutes les phases du stade qui précède. Le tableau suivant présente les trois stades préparatoires et leurs phases respectives.

Le modèle de Gordon (2003) montre que, de zéro à trois ans, l'enfant s'engage dans un stade d'acculturation. Ses réponses à la musique sont d'abord spontanées, puis s'adaptent au contexte lorsqu'il tente d'établir une relation entre ses mouvements, son babillage et les sons musicaux de l'environnement.

Au moment où l'enfant atteint le stade imitatif (deux-cinq ans), il réalise que ses mouvements et son babillage ne sont pas synchronisés avec les sons musicaux de l'environnement. Il affine alors ses capacités découte et devient plus attentif à l'exactitude de la reproduction musicale. Néanmoins, le soutien constant d'un parent ou d'un éducateur est requis, car les capacités cognitives de l'enfant demeurent limitées. En restreignant les motifs mélodiques et rythmiques produits par l'enfant, l'adulte crée, chez l'apprenant, un conflit cognitif qui amène ce dernier à revoir sa conception du système musical. Cet aspect, directement inspiré de la théorie vygotskienne, incite l'enfant à développer de nouvelles stratégies lui permettant de reproduire avec exactitude les enchaînements musicaux produits par son entourage.

6 Dans le présent contexte, il serait plus approprié d'employer le terme capacité que celui d'aptitude. Cependant, Gordon ne distingue pas ces deux termes et privilégie le second dans ses travaux.

7 Selon Gordon (2003), l'audiation est un processus cognitif par lequel le cerveau attribue une signification aux sons musicaux. 
Tableau 1. Stades préparatoires et phases développementales (Gordon, 2003)

\begin{tabular}{|l|l|}
\hline \multicolumn{1}{|c|}{ Stades préparatoires* } & \multicolumn{1}{c|}{ Phases développementales } \\
\hline \multirow{5}{*}{$\begin{array}{l}\text { Acculturation (0-3 ans) } \\
\text { Découverte semi-consciente de } \\
\text { son environnement musical }\end{array}$} & $\begin{array}{l}\text { Éveil: } \\
\text { L'enfant écoute attentivement les sons musicaux de } \\
\text { l'environnement. }\end{array}$ \\
\cline { 2 - 3 } & $\begin{array}{l}\text { Réponses spontanées: } \\
\text { Les mouvements et le babillage de l'enfant sont intuitifs et non } \\
\text { cohérents en réponse aux sons musicaux de l'environnement. }\end{array}$ \\
\cline { 2 - 3 } $\begin{array}{l}\text { Imitation (2-5 ans) } \\
\text { Exploration consciente de son } \\
\text { environnement musical } \\
\text { L'enfant tente d'établir une relation entre ses mouvements, son } \\
\text { babillage et les sons musicaux de l'environnement. }\end{array}$ \\
\hline \multirow{5}{*}{$\begin{array}{l}\text { Assimilation (4-6 ans) } \\
\text { Prise de conscience de soi en rap- } \\
\text { port avec l'environnement musical }\end{array}$} & $\begin{array}{l}\text { Abandon de l'égocentrisme: } \\
\text { L'enfant reconnalt que ses mouvements et son babillage ne sont } \\
\text { pas synchronisés avec les sons musicaux de l'environnement. }\end{array}$ \\
\cline { 2 - 3 } & $\begin{array}{l}\text { Socialisation: } \\
\text { L'enfant imite avec une certaine précision les sons musicaux } \\
\text { de son environnement, particulièrement les motifs tonals et } \\
\text { rythmiques. }\end{array}$ \\
\hline & $\begin{array}{l}\text { Introspection: } \\
\text { L'enfant reconnalt son manque de coordination entre son chant, } \\
\text { sa respiration et ses mouvements corporels. }\end{array}$ \\
\hline $\begin{array}{l}\text { Coordination: } \\
\text { L'enfant coordonne son chant avec sa respiration et ses mouve- } \\
\text { ments corporels. }\end{array}$ \\
\hline
\end{tabular}

*Traduction libre de Gordon (2003)

Lorsqu'il s'engage dans le stade d'assimilation (de quatre à six ans), l'enfant prend graduellement conscience de l'absence de coordination entre ses productions vocales, sa respiration et ses mouvements corporels. Gordon (2003) reconnaît que l'enfant doit être capable de les coordonner pour ensuite pouvoir interagir musicalement et de façon cohérente avec d'autres personnes. Dès que la phase de coordination est maîtrisée, l'enfant commence son apprentissage musical formel par l'intermédiaire des six stages of audiation. Il atteint généralement le premier de ces stades vers lâge de six ans.

Gordon $(1988,1999,2003)$ croit que, dès sa naissance, l'enfant est un " être » musical et que les facteurs innés et environnementaux influencent le développement de ses aptitudes en musique. Il établit un parallèle avec le langage en montrant que le babillage permet à l'enfant de communiquer avec son entourage en utilisant des séquences qui découlent directement de sa langue maternelle (de Boysson-Bardies 2005). L'enfant perçoit d'abord les mots, puis il les imite et enfin, il produit de courts énoncés. En musique, le babillage tonal et le babillage rythmique jouent un rôle semblable, puisque les deux développent les capacités d'écoute, de création et de communication. Selon le chercheur, chaque parent a le devoir d'initier son enfant à la musique avant que commencent les apprentissages formels à l'école.

Le modèle de Gordon (2003) éclaire le développement des capacités mélodiques et des capacités rythmiques au cours des premières années de vie. Néanmoins, le chercheur reconnaît lui-même le caractère "sommaire " de son modèle en affirmant qu'il brosse un portrait général du développement musical 
de l'enfant avant que celui-ci n'aborde les six stages of audiation. Ses fondements théoriques rejoignent ceux de Vygotsky (1978) et ceux de Piaget (1977), mais certains auteurs critiquent son appropriation du concept d'assimilation (Stokes 1996; Woodford 1996). Piaget (1977) ne considère pas que l'assimilation soit un stade, mais pense qu'il s'agit plutôt d'un pôle de développement mental qui se retrouve à tous les stades du développement (comme l'accommodation, d'ailleurs). Une certaine confusion terminologique affecte donc la validité interne du modèle de Gordon (2003). Malgré tout, le travail du chercheur mérite dêtre souligné. Gordon (1999) insiste sur l'importance que l'enfant conserve un bon niveau d'aptitudes musicales pour pouvoir comprendre, apprécier et interpréter la musique à un âge plus avancé. Une exposition à un environnement musical riche durant la petite enfance est essentielle afin de nourrir cet intérêt.

\section{LE MOdÈLE DE HARGREAVES}

Le modèle de Hargreaves (1995) résume la pensée de nombreux psychologues de la musique qui se sont intéressés au développement des capacités à chanter, de la représentation graphique, de la perception mélodique et de la composition. Le chercheur a rassemblé sous la forme d'un tableau les principales caractéristiques permettant déclairer le développement musical normatif et expert.

Hargreaves précise que le développement musical normatif et le développement musical expert sont associés respectivement aux capacités innées de l'enfant et à l'apprentissage. Selon lui, le développement normatif est défini comme " le développement dont témoignent naturellement - donc en l'absence de toute attention ou information spécialisée - les enfants au cours de leur croissance au sein d'une culture donnée ", alors que le développement expert renvoie au " développement de hauts niveaux d'aptitudes musicales [...] et est dispensé par un corps professoral conscient des objectifs particuliers à atteindre " (1995, 174). Le modèle que propose Hargreaves s'intéresse principalement au développement musical normatif. Celui-ci comporte cinq phases développementales, inspirées de la théorie piagétienne.

Les deux premières phases du tableau, celles de la petite enfance (0-5 ans), se rapprochent des idées de Piaget (1977). La première phase (0-2 ans) porte d'ailleurs la même appellation. Il s'agit d'une période où l'enfant explore et tente de s'adapter progressivement à son environnement par des activités sensorielles et motrices. La deuxième phase (2-5 ans) est caractérisée par la représentation figurale. Comme le mentionne Zulauf (2002), " [...] l'accès à cette phase intervient par l'avènement de la fonction symbolique, ce qui implique la capacité, nouvelle chez le petit, de concevoir des objets en dehors de leur présence physique $^{8}$. "C'est lors de la troisième phase (5-8 ans) que l'enfant développe ses conventions musicales. Par procédés imitatifs, il tente de reproduire la musique comme le fait l'adulte. Il développe également un système de représentation musicale personnel qu'il affinera progressivement en découvrant les diverses règles du système tonal. La quatrième phase correspond à l'apprentissage formel de la musique alors que le préadolescent mobilise l'ensemble de ses capacités musica-

8 Madeleine Zulauf. 2002. Loc. Cit, 41. 
les. La cinquième phase renvoie au développement expert. Elle n'est atteinte que par une minorité d'individus ayant des habiletés musicales remarquables.

Le travail de synthèse effectué par Hargreaves est certes intéressant, mais la classification qu'il propose est-elle représentative du développement musical de l'enfant? À l'instar de Zulauf (2002), nous nous demandons pourquoi, à la phase sensori-motrice, « la danse rythmique " est classée dans la colonne Chant, alors qu'elle a peu de rapports avec la production vocale. Qui plus est, dans la dernière colonne, manifestement inspirée du modèle de Swanwick et Tillmann (1986), " l'assimilation de la musique à la culture » ne devrait-elle pas figurer également à la ligne sensori-motrice? À cet égard, des recherches montrent que dès les premiers mois de sa vie, le bébé produit des séquences sonores (babillage) qui sont directement influencées par sa découverte du système musical tonal (Trehub 2003). Hargreaves et North (2001) sont d'avis que le tableau pourrait être modifié et que d'autres catégories pourraient s'y ajouter.

Tableau 2. Modèle de Hargreaves (1995)

\begin{tabular}{|c|c|c|c|c|}
\hline Phases & Chant & $\begin{array}{l}\text { Représentation } \\
\text { graphique }\end{array}$ & $\begin{array}{l}\text { Perception } \\
\text { mélodique }\end{array}$ & Composition \\
\hline $\begin{array}{l}\text { Professionnelle } \\
\text { (15 ans et plus) }\end{array}$ & & & & $\begin{array}{l}\text { Stratégies de jeu } \\
\text { et réflexive }\end{array}$ \\
\hline $\begin{array}{l}\text { Système de règles } \\
\text { (8-15 ans) }\end{array}$ & $\begin{array}{l}\text { intervalles, } \\
\text { échelles }\end{array}$ & $\begin{array}{l}\text { formelle, } \\
\text { métrique }\end{array}$ & $\begin{array}{l}\text { reconnaissance } \\
\text { analytique des } \\
\text { intervalles, } \\
\text { stabilité tonale }\end{array}$ & $\begin{array}{l}\text { conventions } \\
\text { idiomatiques }\end{array}$ \\
\hline $\begin{array}{l}\text { Schématique } \\
\text { (5-8 ans) }\end{array}$ & $\begin{array}{l}\text { première } \\
\text { ébauche de } \\
\text { chansons }\end{array}$ & $\begin{array}{l}\text { figurale-metrique, } \\
\text { plus d'une dimen- } \\
\text { sion }\end{array}$ & $\begin{array}{l}\text { conservation } \\
\text { des propriétés } \\
\text { mélodiques }\end{array}$ & $\begin{array}{l}\text { conventions } \\
\text { vernaculaires }\end{array}$ \\
\hline $\begin{array}{l}\text { Figurale } \\
\text { (2-5 ans) }\end{array}$ & $\begin{array}{l}\text { " grandes lig- } \\
\text { nes » des chan- } \\
\text { sons: fusion entre } \\
\text { le chant spontané } \\
\text { et les chansons } \\
\text { de la culture }\end{array}$ & $\begin{array}{l}\text { figurale: une } \\
\text { seule dimension }\end{array}$ & $\begin{array}{l}\text { caractéristiques } \\
\text { globales: hauteur, } \\
\text { contour }\end{array}$ & $\begin{array}{l}\text { Assimilation de } \\
\text { la musique à la } \\
\text { culture }\end{array}$ \\
\hline $\begin{array}{l}\text { Sensori-motrice } \\
\text { (0-2 ans) }\end{array}$ & $\begin{array}{l}\text { babillage, danse } \\
\text { rythmique }\end{array}$ & $\begin{array}{l}\text { griffonnage: } \\
\text { "equivalent } \\
\text { d'action " }\end{array}$ & $\begin{array}{l}\text { reconnaissance } \\
\text { des contours } \\
\text { mélodiques }\end{array}$ & $\begin{array}{l}\text { sensorielle, } \\
\text { manipulation }\end{array}$ \\
\hline
\end{tabular}

\section{CONCLUSION}

En somme, il est clair que les cinq modèles analysés et critiqués précédemment permettent d'établir les grandes lignes du développement musical chez l'enfant d'âge préscolaire. En s'inspirant des concepts de Piaget et de Vygotsky, les travaux de chercheurs tels que Sloboda, Swanwick et Tillmann, Zenatti, Gordon et Hargreaves montrent lincidence directe du capital biologique et du milieu socioculturel sur lémergence des savoirs musicaux. Selon eux, il apparait que la place et la valeur accordées à léducation musicale durant la petite enfance - jumelées au potentiel unique de chaque enfant - contribuent significativement au développement global des jeunes apprenants. A cet égard, plusieurs travaux récents ont mis en valeur l'effet bénéfique de l'éducation musicale sur 
d'autres types d'apprentissages. Sur le plan socioaffectif, des études révèlent que les activités musicales facilitent l'émergence de capacités relatives à la communication, rehaussent l'estime de soi et permettent, à l'aide des pratiques employées en musicothérapie, d'harmoniser les relations interpersonnelles chez les élèves présentant des difficultés d'intégration sociale (Gourgey, 1998). D’autres études indiquent que léducation musicale contribue également au développement des capacités kinesthésiques (Sidnell 1986) et des capacités cognitives au cours de la petite enfance et de l'enfance (Radocy et Boyle 2003). Qui plus est, de nombreux chercheurs soutiennent également que l'éducation musicale peut améliorer l'apprentissage des autres matières scolaires et ainsi rehausser le rendement pédagogique. Des travaux réalisés auprès d'élèves du primaire ont montré l'impact positif significatif de l'apprentissage de la musique sur l'étude des mathématiques (Hetland 2000; Vaughn 2000), de la langue première (Bolduc 2006; Douglas et Willatts 1994; Ribière-Raverlat 1997) et de la langue seconde (Dominguez 1991; de Frece 1995; Lowe 1995; 1998).

Dans une perspective plus générale, nous sommes d'avis que la musique peut s'intégrer habilement à une approche pédagogique interdisciplinaire en favorisant l'engagement scolaire de l'élève, en renforçant son sentiment identitaire et en rehaussant, de surcroît, son rendement scolaire général (Lowe 1995; 1998). Il est clair que l'enseignement interdisciplinaire peut aider à relier et contextualiser les apprentissages afin de favoriser l'acquisition de connaissances diverses (Lataille-Démoré 1998). Toutefois, cela ne doit pas nuire à l'acquisition par le jeune élève des concepts et des contenus propres à chaque discipline. Les notions de base acquises dans chaque domaine sont elles aussi primordiales et l'apprentissage peut difficilement être global et significatif si la formation de lélève souffre de lacunes intradisciplinaires.

Pour pouvoir réaliser des projets de nature interdisciplinaire jumelant les différentes matières scolaires avec la musique, les enseignants généralistes doivent absolument posséder des notions approfondies dans ce domaine. Leur formation doit viser non seulement l'acquisition des connaissances liées à la théorie musicale, mais également l'acquisition d'habiletés musicales et de concepts relatifs au développement musical durant la petite enfance et l'enfance. Il est donc impératif d'augmenter le nombre de cours consacrés à léducation musicale dans les facultés des sciences de l'éducation pour assurer le développement professionnel des futurs titulaires de classe au préscolaire et au primaire.

Ces considérations ne minimisent en rien l'importance du rôle que doit assurer l'enseignant spécialiste en éducation musicale au préscolaire et au primaire. Au contraire, nous sommes persuadés que celui-ci demeure la personne la mieux placée pour assurer une éducation musicale de qualité au sein de la classe. Cela dit, si on veut lui donner aussi la possibilité de réaliser des projets de nature interdisciplinaire, il faudrait lui fournir une formation adéquate. La formation initiale des éducateurs musicaux est trop souvent centrée sur leur spécialité, au détriment de l'acquisition de compétences dans d’autres domaines d'apprentissage. Il suffirait de décloisonner l'apprentissage et d'offrir plus de cours sur l'interdisciplinarité scolaire pour sensibiliser les éducateurs musicaux à l'importance de cette approche. 
Tout bien pesé, nous devons réitérer l'importance qu'il faut accorder aux recherches touchant l'émergence des capacités musicales au cours de la petite enfance. Les premières années de vie sont cruciales pour le développement d'habiletés que l'individu sollicitera tout au long de son existence. Il est impératif que les parents et les éducateurs prennent conscience du rôle que doit occuper l'éducation musicale dans la vie de leur progéniture.

\section{RÉFÉRENCES}

Bernstein, Leonard. 1976. The unanswered question: Six talks at Harvard. Cambridge: Harvard University Press.

Bolduc, Jonathan. 2006. Les effets d'un programme d'entraînement musical expérimental sur l'appropriation du langage écrit à la maternelle. Thèse de doctorat inédite, Québec: Université Laval.

Bringuier, Jean.-Claude. 1977. Conversations libres avec Jean Piaget. Paris: Laffont.

Chauveau, Gérard. 1997. Comment l'enfant devient lecteur. Paris: Retz.

Chomsky, Noam. 1957. Syntactic structures. Mouton: La Haye.

- 1965. Aspects of the theory of syntax. Cambridge: MIT Press.

Cloutier, Richard, Gosselin, Pierre et Tap, Pierre, $2^{\mathrm{e}}$ éd. 2004. Psychologie de lenfant. Boucherville: Gaétan Morin.

de Boysson-Bardies, Bénédicte, $2^{\mathrm{e}}$ éd. 2005. Comment la parole vient aux enfants. Paris: Odile Jacob.

de Frece, Robert J. 1995. "The relationship of french immersion education to tonal skills of first and fourth grade canadian children ». Canadian journal of research in music education, 35(7): 10-16.

Deliège, Irène, et Sloboda, John A, éd. 1995. Naissance et développement du sens musical. Paris: Presses universitaires de France.

Dominguez, Dora S. 1991. Developing language through a musical program and its effect on the reading achievement of spanish-speaking migrant children. Thèse de doctorat inedited, Kalamazoo: Western Michigan University.

Douglas, Shelia, et Willatts, Peter. 1994. « The relationship between musical ability and literacy skills ». Journal of research in reading, 17(2): 99-107.

Fraisse, Paul. 1974. Psychologie du rythme. Paris: Presses universitaires de France.

Francès, Robert, $2^{\mathrm{e}}$ éd. 1984. La perception de la musique. Paris: Vrin.

Flohr, John W. 2003. Musical lives of young children. Upper Saddle River: Prentice Hall.

Gordon, Edwin E, 1971. The psychology of music teaching. Upper Saddle River: Prentice-Hall.

- 1979. Primary measures of music audiation. Chicago: G.I.A. Publications.

- 1988. Learning sequences in music skill, content and patterns. Chicago: G.I.A. Publications.

- 1999. "All about audiation and music aptitudes ". Music Educator Journal, 86(2): 41-44. 
$3^{\mathrm{e}}$ éd. 2003. A music learning theory for newborn and young children. Chicago: G.I.A. Publications.

Gourgey, Charles. 1998. "Music therapy in the treatment of social isolation in visually impaired children ». Review, 29(4): 157-162.

Green, Michael, et Piel, John A 1989. Theories of human development: A comparative approach. Upper Saddle River: Prentice Hall.

Hargreaves, David J. 1995. "Développement du sens artistique et musical ". In Naissance et développement du sens musical, éd. Irène Deliège et John A Sloboda, 167-197. Paris: Presses universitaires de France.

Hargreaves, David J, et North, Adrian, C. 2001. Musical development and learning: The international perspective. Londres: Continuum.

Hargreaves, David J, et Zimmerman, Marilyn P. 1992. « Developmental theories of music learning $»$. In Handbook of research on music teaching and learning, éd. Robert Colwell, 377-391. New York: Schirmer Books.

Hepper, Peter G. 1991. " An examination of fetal learning before and after birth ». The Irish Journal of Psychology, 12(2): 95-107.

Hetland, Lois. 2000. "Learning to make music enhances spatial reasoning ". Journal of aesthetic education, 34(3-4): 179-238.

Ilari, Beatriz S. 2002. " Music perception and cognition in the first year of life". Early child development and care, 172(3): 311-322.

Imberty, Michel. 1969. Lacquisition des structures tonales chez lenfant. Paris: Klincsieck.

Ivic, Ivan. 1994. « Lev. S. Vygotsky ». Perspectives: revue trimestrielle déducation comparée, 24(3-4): 793-820.

Lataille-Démoré, Diane. 1998. "Le défi de l'intégration des apprentissages et la réforme du programme de formation initiale à l'enseignement en Ontario ». Revue des sciences de l'éducation, 24(1):137-165.

Legendre, Marie-Françoise. 2005. "Lev Vygotsky et le socioconstructivisme en éducation ». In La pédagogie: Théories et pratiques de l'Antiquité à nos jours, $2^{\mathrm{e}}$ éd. Clermont Gauthier et Maurice Tardif, 352-373. Boucherville: Gaétan Morin.

Lowe, Anne. 1995. The effect of the incorporation of music learning into the second-language classroom on the mutual reinforcement of music and language. Thèse de doctorat inédite, Urbana-Champaign: University of Illinois.

—. 1998. "Lintégration de la musique et du français au programme d'immersion française: Avantages pour l'apprentissage des deux matières". Revue des sciences de l'éducation, 24(3): 621-646.

Moog, Helmuth. 1976. The musical experience of the preschool child. Londres: Schott.

Pflederer, Marilyn. 1964. "The responses of children to musical tasks embodying Piaget's principle of conservation. " Journal of research in music education, 12(4): 251-268.

Piaget, Jean, $2^{\mathrm{e}}$ éd. 1961. Les mécanismes perceptifs. Paris: Presses universitaires de France.

— $6^{e}$ éd. 1971. La construction du réel chez l'enfant. Paris: Delachaux et Niestle. 
—. $9^{e}$ éd. 1977. La naissance de l'intelligence chez l'enfant. Paris: Delachaux et Niestle.

. $7^{\mathrm{e}}$ éd. 1978. La formation du symbole chez l'enfant. Paris: Delachaux et Niestle.

- $9^{\mathrm{e}}$ éd. 1984. Le langage et la pensée chez l’enfant. Paris: Denoel.

$\longrightarrow 1^{\mathrm{e}}$ éd. Quadriges. 2003. La représentation du monde chez l'enfant. Paris: Presses universitaires de France.

Piaget, Jean, et Inhelder, B. $4^{\mathrm{e}}$ éd. 1981. La représentation de l'espace chez l'enfant. Paris: Presses universitaires de France.

-19e éd. 2003. La psychologie de l'enfant. Paris: Presses universitaires de France.

Radocy, Rudolf E, et Boyle, David J, $4^{\mathrm{e}}$ éd. 2003. Psychological foundation of music behavior. Springfield: Charles $\mathrm{C}$. Thomas.

Ribière-Raverlat, Jacquotte. 1997. Développer les capacités découte d̀ lécole: Écoute musicale, écoute des langues. Paris: Presses universitaires de France.

Rieber, Robert W, Robinson, David K, et Bruner, Jerome S. 2004. The essential Vygotsky. New York: Kluwer Academic.

Sidnell, Robert G. 1986. «Motor learning in music education. » Psychomusicology, 6: 7-17.

Sloboda, John A. 1985. L'esprit musicien: La psychologie cognitive de la musique. Paris: Mardaga.

Stokes, Ann W. 1996. "Is Edwin Gordon's learning theory a cognitive one? " Philosophy of music education review, 2: 96-108.

Swanwick, Keith. 2001. "Conference keynote: Musical development theories revisited. » Music education research, 3(2): 227-242.

Swanwick, Keith, et Tillmann, June. 1986. "The sequence of musical development: A study of children's composition. " British journal of music education, 3: 305-339.

Thorpe, Leigh A, Trehub, Sandra E, Morrongiello, Barbara A, et Bull, Don. 1988. " Perceptual grouping by infants and preschool children. " Developmental Psychology, 24: 484-491.

Trehub, Sandra E. 2001. " Musical Predispositions in Infancy. " Annals of the New York Academy of Sciences, 930: 3-16.

- 2003. "Foundations: Music perceptions in infancy ". In Musical lives of young children, éd. John W Flohr, 24-29. Upper Saddle River: Prentice Hall.

Vaughn, Kathryn. 2000. « Music and mathematics: Modest support for the oftclaimed relationship." Journal of aesthetic education, 34(3-4): 149-166.

Vygotsky, Lev S. 1978. Mind and society: The development of higher mental processes. Cambridge: Harvard University Press.

Wallon, Philippe. 1996. "Lévolution du dessin de l'enfant. " In Le dessin de l'enfant, éd. Jocelyne Raffier-Malosto, 75-91. Saint-Étienne: La pensée sauvage.

Woodford, Paul G. 1996. " Evaluating Edwin Gordon's music learning theory from a critical thinking perspective. " Philosophy of music education review, 2: 83-95. 
Woodward, Sheila C. 1992, The Transmission Of Music Into The Human Uterus And The Response To Music Of The Human Fetus And Neonate. Thèse de doctorat inédite, Afrique du sud: University of Cape Town.

Zenatti, Arlette. 1967. Perception et intelligence musicales chez l'enfant. Université Paris X-Nanterre, Paris.

. 1976. «Influence de quelques variables socioculturelles sur le développement musical de l'enfant ». Psychologie française, 21(3): 185-190.

_. 1990. "Aspects du développement musical de l'enfant dans l'histoire de la psychologie au XXe siècle ». Les Sciences de léducation, 3-4: 21-38.

Zulauf, Madeleine. 2002. Limites et promesses de quelques théories du développement musical. In $A$ la recherche du développement musical, éd. Martine Wirthner et Madeleine. Zulauf, 19-51. Paris: L'Harmattan

\section{RÉSUMÉ}

Les recherches concernant le développement des capacités musicales chez les jeunes enfants sont en plein essor. Inspirés par différentes théories constructivistes et socioconstructivistes, quelques psychologues de la musique ont, depuis les 30 dernières années, élaboré différents modèles afin déclairer les savoirs musicaux présents à la période préscolaire. Cet article recense, analyse et critique cinq des principaux modèles qui ont été développés au cours de ces trois décennies. Il réitère également l'importance qui doit être accordée à léducation musicale au cours de la petite enfance, et du rôle positif qu'elle peut jouer dans le cadre d'un enseignement interdisciplinaire.

\section{ABSTRACT}

Research on the development of the musical abilities of young children is on the rise. In the past 30 years, music psychologists inspired by constructivist and socioconstructivist theories have developed various approaches in order to better understand the musical knowledge of preschoolers. This article critiques and establishes an inventory of five main trends that have been developed over the last three decades. In addition, it reiterates the importance of music education in the preschool period and the positive role it can play in interdisciplinary curricula. 